\title{
INFLUENCIA DEL DETERIORO DEL CEPILLO DENTAL SOBRE LA HIGIENE ORAL DE LOS ADULTOS MAYORES
}

\author{
${ }^{1}$ Heidy Lorena Tarazona Barbosa, ${ }^{2}$ Sonia Constanza Concha Sánchez, ${ }^{3}$ Gloria Cristina Aránzazu Moya, \\ ${ }^{1}$ Anne Alejandra Hernández Castañeda \\ ${ }^{1}$ Estudiante X semestre F. de Odontología Universidad Santo Tomás, Bucaramanga, Colombia. \\ ${ }^{2}$ Odontóloga U. Santo Tomás, Especialista en Educación y Comunicación para la Salud, MSc Epidemiologia U. Industrial de Santander, \\ Docente Universidad Santo Tomás, Bucaramanga, Colombia. \\ ${ }^{3}$ Odontóloga Universidad Santo Tomás, Especialista en Patología Oral y Medios Diagnósticos U. El Bosque, \\ Docente Universidad Santo Tomás, Bucaramanga, Colombia.
}

Autor responsable de correspondencia: Sonia Constanza Concha Sanchez

Correo electrónico: sococosa@yahoo.com

RESUMEN

Objetivo: Determinar el efecto del deterioro del cepillo dental sobre la higiene oral en la población adulta mayor del centro Bienestar del Anciano Juan Pablo II.

Materiales y métodos: Se realizó un estudio observacional descriptivo de tipo prospectivo de una cohorte de 45 personas de 60 años o más. Se relacionaron variables sociodemográficas y variables clínicas dientes presentes en boca, actitud a la hora del cepillado, índice de cobertura de lengua, índice cuantificado de placa, índice de higiene de prótesis y índice del deterioro del cepillo dental de Rawls y colaboradores, funcionalidad del adulto mediante escala de Barthel. En la prueba estadistica se analizaron las posibles diferencias mediante el test Chi cuadrado o exacto de Fisher para variables cualitativas y la prueba t test para variables cuantitativas.

Resultados: Las reducciones porcentuales en las valoraciones de placa lograda con un cepillo de dientes nuevo fueron significativamente diferentes de los obtenidos con tres meses de uso de los cepillos dentales. Tanto los nuevos y los de 3 meses de uso reduce la placa bacteriana.

Conclusión: entre el deterioro del cepillo y la higiene oral de los adultos mayores no se encontró relación alguna. Por tanto el deterioro del cepillo no parece influenciar en el nivel de higiene oral de los adultos. [Tarazona HL, Concha SC, Aránzazu GC, Hernández AA. Influencia del deterioro del cepillo dental sobre la higiene oral de los adultos mayores. Ustasalud 2010; 9: 89 - 95]

Palabras clave: Adulto mayor, Cepillo dental, Deterioro del cepillo. Higiene oral.

\section{INFLUENCE OF THE TOOTHBRUSH WEAR ON ORAL HYGIENE IN THE ELDERLY}

\section{ABSTRACT}

Objective: To determine the effect of the deterioration of the toothbrush on oral hygiene in the older population Elderly Welfare Center Juan Pablo II.

Methods: A descriptive study of a prospective cohort of 45 people aged 60 or more was done. Se related variables and clinical variables teeth present in mouth behavior when brushing, language coverage rate, quantified plaque index, prosthesis hygiene index and rate of deterioration of Rawls's toothbrush and Col; functionality of the adult Barthel scale. In the test statistic analyzed the potential differences by chi-square test or Fisher exact test qualitative variables and test for quantitative variables.

Results: The percentage reductions in ratings of plaque achieved with a new toothbrush were significantly different from those obtained with three months of using toothbrushes. A both new and 3 month of use reduces plaque.

Conclusion: By observing the deteriorating relationship between tooth brushing and oral hygiene of older adults found no relationship whatsoever. Therefore, the deterioration of the brush does not seem to influence the level of oral hygiene of adults.

Keywords: Elderly, Toothbrush, Wear, Oral hygiene.

ABREVIATURAS: ICP: índice cuantificado de placa, IEHP: índice de higiene de prótesis. 


\section{INTRODUCCIÓN}

El envejecimiento se considera una etapa más del desarrollo evolutivo del ser humano, siendo afectada por cambios fisiológicos los cuales son reflejados en las relaciones sociales, laborales, familiares, lo que contribuye al desarrollo de manifestaciones psicosomáticas a nivel general y oral en el adulto mayor. ${ }^{1}$

Es por esto que el profesional de la salud debe esforzarse por motivar al adulto mayor a mejorar su calidad de vida; enfatizando la necesidad de las personas mayores que residen en centros geriátricos ya que estas son vulnerables y un alto porcentaje presenta condiciones de salud oral bastante precarias; todo lo descrito previamente refleja promover necesidades de brindar mayor atención odontológica, enseñar buenos hábitos de higiene oral, y a la conservación del buen estado del cepillo dental. ${ }^{2} \mathrm{El}$ deterioro del cepillo dental se ve afectado por factores como la destreza del individuo, técnicas de cepillado, el tiempo y la cantidad de veces que este es utilizado, la fuerza ejercida, los hábitos preexistentes, la motivación, la salud mental y capacidad funcional de las personas mayores. Las condiciones reflejadas no solo influyen en el deterioro del cepillo sino que también inciden sobre el nivel de higiene oral de las personas mayores, aspecto que se ve agravado si el cepillo no reúne unas condiciones adecuadas. Los cuales logran desencadenar el aumento de la placa bacteriana produciendo una higiene oral inadecuada, reflejo de una mala higiene oral que puede llegar a desencadenar un aumento en la caries dental y enfermedad periodontal. El cepillado y otros procedimientos de limpieza mecánica son las únicas medidas de prevención y control de la placa bacteriana. ${ }^{3-5}$

Por esto se considera imprescindible que el profesional de odontología enseñe a sus pacientes a sustituir su cepillo de dientes después de presentar una enfermedad o infección por microorganismos, a inspeccionar visualmente el cepillo para que el mismo pueda evaluar el deterioro de este y así el tiempo de vida útil; aunque algunas personas no consideran el recambio del cepillo cuando perciben el apelmazamiento de las cerdas, sino al momento de no sentir que limpia su boca.,

Existe cierta controversia de la efectividad de un cepillo de dientes desgastado, y el uso de un cepillo de dientes nuevo; ya que inclusive, un cepillo de dientes desgastado parece ser efectivo, lo que hace deducir que es más importante la motivación y destreza que tenga el paciente al momento de cepillarse.

No obstante la supervisión del odontólogo y del paciente sobre su cepillo dental no deja de ser importante por las potenciales implicaciones relacio- nadas con la sobre infección aun la falta de efecto del cepillo para remover la placa bacteriana en forma efectiva.

Se ha planteado la posibilidad de que un cepillo de dientes gastado no puede ser un impedimento en la limpieza eficaz de los dientes, lo que es comprobado con un estudio donde se observó que con un mínimo desgaste en el cepillo de dientes, se alcanza el mismo grado de eliminación de placa, que en un cepillo con un desgaste avanzado; 3 por tanto, se puede deducir que en cepillos de dientes con uso mayor a tres meses no son menos eficaces que un cepillo de dientes nuevo, en el proceso de eliminación de placa bacteriana.

Algunos autores reportan que esto no es siempre el caso y que el desgaste del cepillo de dientes es muy variable entre una persona a otra, debido a las diferencias en la forma en que son utilizados los cepillos. ${ }^{3}$

Por lo tanto, el objetivo del presente estudio fue determinar el efecto del deterioro del cepillo dental sobre la higiene oral en la población adulta mayor del centro geriátrico bienestar del anciano Juan Pablo II de Floridablanca.

\section{MATERIALES Y MÉTODOS}

Se realizó un estudio observacional descriptivo de tipo prospectivo de una cohorte de adultos mayores; el universo y la muestra la constituyeron 45 adultos mayores de ambos géneros registrados en el Centro Bienestar del Anciano Juan Pablo II de Floridablanca durante el segundo periodo del 2009.

Se consideraron como criterios de inclusión pacientes adultos de 60 años o más pertenecientes al centro Bienestar del Anciano Juan Pablo II, aptos mentalmente para decidir si aceptaban participar en este estudio, que presentaran dientes naturales en boca o edéntulos totales con prótesis y con capacidad motriz para realizar su cepillado dental. Como criterios de exclusión se definieron, retirados del instituto, con diagnostico de patologías psiquiátricas mayores, alteraciones visuales y con enfermedad severas que les dificultaran realizar sus labores de autocuidado, ni aquellos que rechazaron vincularse a la Investigación.Se creó un formato donde se recolectaron las variables sociodemográficas tales como género, edad, procedencia, nivel de estudio, labor y pensionado. Variables clínicas como dientes presentes en boca, especificando combinada, prótesis fija, coronas, actitud a la hora del cepillado, índice de cobertura de lengua, índice cuantificado de placa (ICP), clasificado en buena, regular y mala; índice de higiene de prótesis clasificado de la misma manera del ICP e índice del deterioro del cepillo dental de Rawls y colaboradores con el cual se ob- 
tienen dos resultados, uno cuantitativo el cual se toma al medir las cerdas de la cabeza del cepillo: Wfs: máxima longitud del cepillo en la parte libre de las cerdas. Was: máxima longitud de las cerdas ancladas a la cabeza del cepillo. Wfe: es la anchura del cepillo en el extremo libre de las cerdas. Wae: es la anchura de las cerdas en el extremo ancladas a la .cabeza del cepillo. Lo: hace referencia a la longitud de las cerdas. Con estas mediciones se aplica la formula que se presenta a continuación:

$\mathrm{WI}=\underline{\mathrm{Wfs}-\mathrm{Was}+\mathrm{Wfe}-\mathrm{Wae}{ }^{*} 100 \%}$

$$
2 \text { *Lo }
$$

El índice cualitativo se representa en una escala:

0 no hay desgaste equivale a un $25 \%$.

1 es desgaste leve entre 26 y $49 \%$.

2 es desgaste medio entre 50 y $75 \%$.

3 es desgaste fuerte entre el 76 y $100 \%{ }^{6}$

Por último la funcionalidad del adulto mediante escala de Barthel analizando actividades de la vida diario, comer, lavarse, vestirse, arreglarse, deposición, micción, usar el retrete, trasladarse, deambular, escalones; siendo clasificado como independiente, dependiente leve, moderado, grave y total.

Se efectuó una prueba piloto en la que se verificó la comprensión de las preguntas y el manejo de los datos, se realizó con cuatro adultos mayores voluntarios que cumplieran con los criterios de inclusión y exclusión ya planteados, con el fin de evaluar la reproducibilidad entre los evaluadores, verificar la utilidad del instrumento y considerar los tiempos requeridos para ejecutar la encuesta; a partir de esta se efectuaron los correctivos necesarios.

Posteriormente, se registraron a los adultos, se les entrego un cepillo de dientes nuevo, se les dio instrucciones de higiene oral después del primer registro de los de datos; se coordinó la aplicación del instrumento en los horarios atención en los meses, octubre, noviembre, diciembre y enero del 2010.

Una vez recolectados los datos se procedió a la sistematización en Excel por duplicado, se verificó la calidad de la digitación mediante la rutina Validate del paquete EPI- INFO 6.04d y se procesó en el paquete estadístico STATA 9.0

Para el plan de análisis, cada una de las variables se describieron mediante medidas de resumen según su naturaleza; para las cuantitativas se obtuvieron promedios y desviaciones estándar y para las cualitativas frecuencias y porcentajes.

En el análisis bivariado se analizaron las posibles diferencias mediante el test ji cuadrado o exacto de
Fisher para variables cualitativas y la prueba t test para variables cuantitativas.

Este estudio se acogió a las normas éticas establecidas por el ministerio de salud en la resolución $\mathrm{N}^{\circ}$ 008430 de 1993 y por el Comité de Ética Institucional.

\section{RESULTADOS}

Se evaluaron 45 participantes 25 hombres 55\% y 20 mujeres $44,4 \%$, de los cuales el $11,1 \%$ no completaron el estudio; el promedio de edad fue de $82,3 \pm$ 6,7 años, el 57,8\% provenía del área rural, el 57,8\% cursó hasta primaria, el 97,8\% desempeñaba alguna labor, el $91,1 \%$ no estaba pensionado y el $82,2 \%$ era funcionalmente independiente (Tabla 1).

En la descripción de la actitud positiva se observa que en la evaluación basal el 75,6\% de los participantes tenía una actitud positiva a la hora del cepillado. En relación con la higiene bucal, el $82,2 \%$ presentaba una cobertura gruesa de placa bacteriana en lengua, el promedio del índice cuantificado de placa fue de $2,5 \pm 0,45$, teniendo en cuenta este índice la mayoría de participantes quedó en la categoría mala higiene con un 95,6\%; por otro lado; el $88,9 \%$ de los participantes usaba prótesis; de éstos en el 55\% los años de vida de la prótesis oscilaron entre 0 y 10 años. Así mismo, el índice de control de placa en caries mostró un promedio de 0,95 $\pm 0,14$ y mostró que el $97,5 \%$ de los participantes tenía una mala higiene en la prótesis (Tabla 2).

Se observo que a lo largo del periodo de seguimiento el promedio del índice de deterioro va aumentando, tanto en el grupo de deterioro bajo como el grupo de deterioro alto del cepillo; siendo mayor en el grupo de deterioro alto (Tabla 3).

En relación con la actitud hacia el cepillado, la mayoría de los participantes tuvo una actitud positiva durante todo el periodo de estudio. El menor porcentaje de participantes con actitud positiva se presentó en la evaluación basal con un porcentaje de $72,5 \%$, sin embargo, este porcentaje aumentó al primer mes y se mantuvo hasta el final del seguimiento con porcentajes de 95\% (Tabla 2).

Por otro lado, un elevado porcentaje de participantes presentó durante todo el seguimiento una cobertura gruesa de placa bacteriana en lengua, los resultados globales muestran porcentajes por encima del $60 \%$ para todas las evaluaciones. Sin embargo, no se observa una tendencia clara a la disminución de la placa bacteriana en lengua puesto que en el mes 1 y 2 la higiene en lengua fue significativamente menor comparado con la evaluación previa; no obstante, en el mes tres ocurrió un aumento significativo de la placa comparado con el mes previo $(72,5 \%$ vs 60\%) (Tabla 2). 
Se encontró que el promedio del índice cuantificado de placa fue disminuyendo durante el seguimiento, las tres evaluaciones fueron menores comparadas con la evaluación basal, tanto para el grupo de deterioro bajo como para el grupo de deterioro alto, con una diferencia estadísticamente significativa en todos los casos, excepto para la última evaluación (Figura 1).

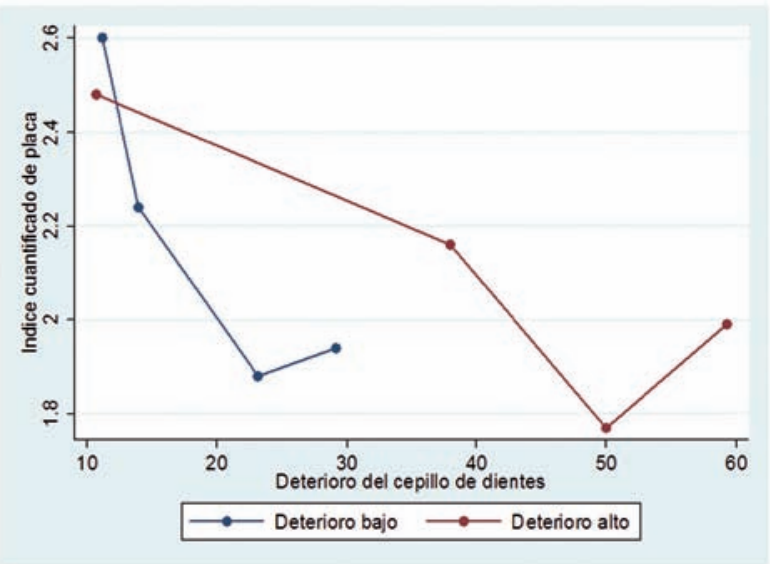

Figura 1. Distribución del promedio del índice cuantificado de placa en las cuatro evaluaciones
En el grupo de deterioro bajo el promedio del índice para evaluar higiene de prótesis fue significativamente menor en la evaluación realizada en el mes dos comparado con la evaluación previa y la evaluación basal; a su vez significativamente mayor comparado con la evaluación en el mes 3. En el grupo de deterioro alto se observa un comportamiento similar al del grupo liviano. No se observaron diferencias estadísticamente significativas por grupo de uso (Tabla 3).

Se observo que durante el período de seguimiento la higiene en boca aumentó puesto que en las tres evaluaciones los promedios de las diferencias del índice cuantificado de placa fueron significativamente menores comparadas con la evaluación basal, excepto para el tercer mes. Mientras que la higiene en prótesis no se modificó substancialmente, puesto que solo en el mes dos el promedio del índice para evaluar la higiene en prótesis fue significativamente menor comparado con la evaluación basal.

Las características mostraron que el $82,2 \%$ eran personas funcionalmente independiente. El 13,4\% dependientes leves, el 2,2\% dependientes moderados y un 2,2 dependientes totales (Tabla 1).

Tabla 1. Características sociodemográficas basales de la población de estudio, según el uso del cepillo de dientes.

\begin{tabular}{|c|c|c|c|c|}
\hline Variable & $\begin{array}{c}\text { Deterioro Bajo } \\
25(55,5)\end{array}$ & $\begin{array}{c}\text { Deterioro } \\
\text { Alto } 15(33,3)\end{array}$ & $\begin{array}{c}\text { Perdidos } \\
5(11,1)\end{array}$ & $\begin{array}{c}\text { Total } \\
45(100)\end{array}$ \\
\hline \multicolumn{5}{|l|}{ Sexo n (\%) } \\
\hline Femenino & $10(40)$ & $9(60)$ & $1(20)$ & $20(44,4)$ \\
\hline Masculino & $15(60)$ & $6(40)$ & $4(80)$ & $25(55,6)$ \\
\hline Edad $\bar{x} \pm D E$ & $83,04 \pm 6,7$ & $80,4 \pm 7,5$ & $84,6 \pm 4,1$ & $82,3 \pm 6,7$ \\
\hline \multicolumn{5}{|l|}{ Procedencia n (\%) } \\
\hline Urbano & $11(44)$ & $6(40)$ & $2(40)$ & $19(42,2)$ \\
\hline Rural & $14(56)$ & $9(60)$ & $3(60)$ & $26(57,8)$ \\
\hline \multicolumn{5}{|l|}{ Nivel de estudios n (\%) } \\
\hline Ninguno & $7(28)$ & $4(26,7)$ & $2(40)$ & $13(28,9)$ \\
\hline Primaria & $14(56)$ & $10(66,6)$ & $2(40)$ & $26(57,8)$ \\
\hline Secundaria & $4(16)$ & $1(6,7)$ & $1(20)$ & $6(13,3)$ \\
\hline \multicolumn{5}{|l|}{ Labor n (\%) } \\
\hline $\mathrm{Si}$ & $24(96)$ & $15(100)$ & $5(100)$ & $44(97,8)$ \\
\hline No & $1(4)$ & $0(0)$ & $0(0)$ & $1(2,2)$ \\
\hline \multicolumn{5}{|l|}{ Pensionado n (\%) } \\
\hline No & $23(92)$ & $14(93,3)$ & $4(80)$ & $41(91,1)$ \\
\hline $\mathrm{Si}$ & $2(8)$ & $1(6,7)$ & $1(20)$ & $4(8,9)$ \\
\hline \multicolumn{5}{|l|}{ Capacidad funcional } \\
\hline Independiente & $19(76)$ & $14(93,3)$ & $4(80)$ & $37(82,2)$ \\
\hline Dependiente leve & $5(20)$ & $1(6,7)$ & $0(0)$ & $6(13,4)$ \\
\hline Dependiente moderado & $0(0)$ & $0(0)$ & $1(20)$ & $1(2,2)$ \\
\hline Dependiente total & $1(4)$ & $0(0)$ & $0(0)$ & $1(2,2)$ \\
\hline
\end{tabular}


Tabla 2. Actitud hacia el cepillado y e higiene bucal basal de la población de estudio, según el uso del cepillo de dientes.

\begin{tabular}{|c|c|c|c|c|}
\hline Variable & $\begin{array}{c}\text { Deterioro Bajo } \\
25(55,5)\end{array}$ & $\begin{array}{c}\text { Deterioro Alto } \\
15(33,3)\end{array}$ & $\begin{array}{c}\text { Perdidos } \\
5(11,1)\end{array}$ & $\begin{array}{c}\text { Total } \\
45(100)\end{array}$ \\
\hline \multicolumn{5}{|l|}{ Actitud a la hora del cepillado $\mathrm{n}(\%)$} \\
\hline Positiva & $18(72)$ & $11(73,3)$ & $0(0)$ & $11(24,4)$ \\
\hline Negativa & $7(28)$ & $4(26,7)$ & $5(100)$ & $34(75,6)$ \\
\hline \multicolumn{5}{|l|}{ Cobertura de la placa bacteriana en lengua n (\%) } \\
\hline Sin cobertura & $2(8)$ & $1(6,7)$ & $0(0)$ & $3(6,7)$ \\
\hline Delgada & $4(16)$ & $1(6,7)$ & $0(0)$ & $5(11,1)$ \\
\hline Gruesa & $19(76)$ & $13(86,6)$ & $5(100)$ & $37(82,2)$ \\
\hline Índice cuantificado de placa $\bar{x} \pm D E$ & $2,6 \pm 0,52$ & $2,5 \pm 0,32$ & $2,4 \pm 0,35$ & $2,5 \pm 0,45$ \\
\hline Regular & $2(8)$ & $0(0)$ & $0(0)$ & $2(4,4)$ \\
\hline Mala & $23(92)$ & $15(100)$ & $5(100)$ & $43(95,6)$ \\
\hline \multicolumn{5}{|l|}{ Dientes presentes n (\%) } \\
\hline Naturales & $3(12)$ & $2(13,3)$ & $0(0)$ & $5(11,1)$ \\
\hline Prótesis total & $16(64)$ & $12(80)$ & $3(60)$ & $31(68,9)$ \\
\hline Prótesis y naturales & $6(24)$ & $1(6,7)$ & $2(40)$ & $9(20)$ \\
\hline \multicolumn{5}{|l|}{ Tiempo control de placa bacteriana en prótesis n (\%) } \\
\hline $0-10$ años & $14(63,6)$ & $6(46,2)$ & $2(40)$ & $22(55)$ \\
\hline 10-20 años & $4(18,2)$ & $1(7,6)$ & $2(40)$ & $7(17,5)$ \\
\hline$>20$ años & $4(18,2)$ & $6(46,2)$ & $1(20)$ & $11(27,5)$ \\
\hline Índice control de placa bacteriana en prótesis $\bar{x} \pm \mathrm{DE}$ & $0,94 \pm 0,17$ & $0,94 \pm 0,12$ & $0,99 \pm 0,02$ & $0,95 \pm 0,14$ \\
\hline Regular & $1(92)$ & $0(0)$ & $0(0)$ & $1(2,5)$ \\
\hline Malo & $21(8)$ & $13(100)$ & $5(100)$ & $39(97,5)$ \\
\hline
\end{tabular}

Tabla 3. Distribución del deterioro del cepillo dental de acuerdo con el uso del mismo durante el seguimiento.

\begin{tabular}{|c|c|c|c|c|c|}
\hline Grupo & Evaluación basal & Mes 1a & Mes 2a & Mes 3a & $\mathbf{P}^{*}$ \\
\hline $\begin{array}{l}\text { Deterioro Bajo } \\
\text { Índice de deterioro } \overline{\mathrm{x}} \pm \mathrm{DE} \\
\text { Categorías } \mathrm{n}(\%)\end{array}$ & $11,2 \pm 8,3^{\text {bcd }}$ & $14 \pm 8,7$ bce & $23,2 \pm 11,8$ bde & $29,2 \pm 9,1^{\text {cde }}$ & $(\mathrm{p}<0,05)$. \\
\hline Ningún desgaste & $23(92)$ & $23(92)$ & $13(52)$ & $9(36)$ & \\
\hline Desgaste bajo & $2(8)$ & $2(8)$ & $12(48)$ & $16(64)$ & \\
\hline Desgaste medio & $0(0)$ & $0(0)$ & $0(0)$ & $0(0)$ & \\
\hline Desgaste alto & $0(0)$ & $0(0)$ & $0(0)$ & $0(0)$ & \\
\hline $\begin{array}{l}\text { Deterioro Alto } \\
\text { Índice de deterioro } \overline{\mathrm{x}} \pm \mathrm{DE} \\
\text { Categorías } \mathrm{n}(\%)\end{array}$ & $10,7 \pm 2,6^{\mathrm{bcd}}$ & $38 \pm 24,8^{\text {bce }}$ & $50 \pm 14,6$ bde & $59,3 \pm 17,5^{\text {cde }}$ & $(\mathrm{p}<0,05)$. \\
\hline Ningún desgaste & $15(100)$ & $4(26,7)$ & $0(33,3)$ & $0(0)$ & \\
\hline Desgaste bajo & $0(0)$ & $7(46,7)$ & $5(33,3)$ & $0(0)$ & \\
\hline Desgaste medio & $0(0)$ & $2(13,3)$ & $9(60)$ & $12(80)$ & \\
\hline Desgaste alto & $0(0)$ & $2(13,3)$ & $1(6,7)$ & $3(20)$ & \\
\hline $\begin{array}{l}\text { Global } \\
\text { Índice de deterioro } \overline{\mathrm{x}} \pm \mathrm{DE} \\
\text { Categorías } \mathrm{n}(\%)\end{array}$ & $11,1 \pm 6,5^{\mathrm{bcd}}$ & $22 \pm 19,5^{\text {bce }}$ & $29,9 \pm 19,8$ bde & $40,5 \pm 19,5^{\text {cde }}$ & $(\mathrm{p}<0,05)$. \\
\hline Ningún desgaste & $38(95)$ & $27(67,5)$ & $13(32,5)$ & $9(22,5)$ & \\
\hline Desgaste bajo & $2(5)$ & $9(22,5)$ & $17(42,5)$ & $16(40)$ & \\
\hline Desgaste medio & $0(0)$ & $2(5)$ & $9(22,5)$ & $12(30)$ & \\
\hline Desgaste alto & $0(0)$ & $2(5)$ & $1(2,5)$ & $3(7,5)$ & \\
\hline
\end{tabular}

*Prueba estadística t de Student. 


\section{DISCUSIÓN}

La higiene bucal es parte integral de la higiene personal: un pilar que promueve la salud del individuo; para lograr esto debe garantizarse un buen cepillado, dirigido a una correcta remoción de la placa dentobacteriana y restos de sustancias consumidas. ${ }^{5}$

El objetivo de la presente investigación fue evaluar si el deterioro del cepillo dental tiene efecto sobre la eliminación de la placa bacteriana en el adulto mayor en el cual se evaluaros factores como la actitud del adulto ante el cepillado, índice de capacidad funcional y niveles de placa bacteriana.

La higiene oral debe desarrollarse con técnicas de intervención educativa, capaces de modificar conocimientos inadecuados y proporcionarles la motivación necesaria para que cuiden su propia salud, la de su familia y demás miembros de la comunidad. Se ha sugerido que pacientes bien motivados presentan elevado interés en la educación y ejecución de técnicas de higiene oral, como la mejora en la técnica de cepillado. ${ }^{7-9}$

Se observo que los participantes que mostraron actitud positiva ante el estudio $(72,5 \%)$ aumentaron a un $95 \%$ que se mantuvo hasta el final. El cepillado dental, por ser el método más difundido y contar con un alto grado de aceptabilidad social, se recomienda que sea incorporado como parte fundamental para mantener la salud bucal; sin embargo, en la mayoría de las personas los procedimientos habituales de higiene bucal no logran controlar la placa dentobacteriana. ${ }^{10-14}$

Tan y Daly (2002) exponen que los cepillos de dientes con un mínimo de desgaste alcanzado puede eliminar el mismo nivel de placa dentobacteriana que la que es eliminada con un cepillo dental con máximo desgaste, probado con porcentajes de reducción de placa con el uso de cepillos nuevos de $43.1 \%$ y para los cepillos con tres meses de uso $42.0 \%{ }^{3}$

Disminuyó tanto en el grupo de deterioro bajo como en el grupo de deterioro alto a medida que el deterioro del cepillo aumenta; participantes con un deterioro del cepillo alto su ICP era de $26,7 \%$ comparado con el examen inicial el cual fue de $100 \%$ mala higiene oral.

El Tercer Estudio Nacional de Salud Bucal (ENSAB III) concluyó que en las personas mayores de 20 años se produce una leve reducción en el porcentaje de placa dentobacteriana, la reducción en las personas de edad avanzada estaba influida por la pérdida dentaria. Cifras estadísticas muestran que personas con edades comprendidas entre 55 a 59 años presentan un porcentaje del 47,3\% de placa dentobacteriana; entre 60 a 64 años un $32,7 \%$ y mayores de 65 años $26,9 \%{ }^{12}$
En este estudio se encontró una diferencia significativa en la medida inicial del ICP que fue de un 95,6\% catalogado como mala higiene oral y en el tercer mes fue de $37.5 \%$ lo que se aproxima a los porcentajes encontrados en el ENSAB III.

Rangel y colaboradores plantean que el crecimiento de la población envejecida ha producido un número creciente de personas que requieren prótesis dentales. Los microporos que aparece en el acrílico de las prótesis proporcionan una amplia gama de ambientes para el desarrollo de microorganismos que pueden amenazar la salud de un paciente fisicamente vulnerable. El mantenimiento de las prótesis es importante para la salud del paciente y para mantener la estética. Estrategias mecánicas, químicas y una combinación de ambas, están disponibles para los pacientes con el objetivo de facilitar la higiene de las prótesis. Los profesionales dentales deben tener un conocimiento actualizado acerca de estrategias para la limpieza de las prótesis, con el fin de aumentar al máximo la calidad del servicio ofrecido a los pacientes portadores de estas. ${ }^{13}$ El estudio no mostro una mejora en la higiene de prótesis ya que el promedio de placa en la medida inicial es de 0,95 y en el tercer mes de estudio no encontramos diferencias significativas con un promedio de 0,92 de placa presente en prótesis.

Con lo que podemos concluir las razones para que el índice de higiene de prótesis no disminuyera, es el estado de la prótesis dental ya que un $46 \%$ de los sujetos tiene un porcentaje de vida con sus prótesis de más de 20 años.

Bergstrom (1973), y Tan y Daly (2002) sugieren que la variación en cantidad de desgaste sufrido por los cepillos de dientes durante su uso puede ser causado por las distintas fuerzas y las técnicas de cepillado de los dientes, los pacientes que se cepillan con la cantidad de fuerza más alta producirá mayor desgaste del cepillo de dientes. ${ }^{3,4}$ Lo que se puede sugerir es que probablemente las diferencias en la fuerza y técnica de cepillado hacen que el deterioro en el cepillo de dientes sea diferente y por lo tanto se identifiquen dos grupos: deterioro alto y bajo. ${ }^{3}$

Durante el seguimiento del deterioro del cepillo se observo un incremento en el promedio acorde con el uso dado a lo largo del estudio, se encontró en la primera medición del deterioro del cepillo un promedio de 11,1 en la evaluación basal y de 40,5 en la última evaluación.

Se puede concluir que el deterioro del cepillo es mayor en hombres que en las mujeres del Centro Bienestar del Anciano Juan Pablo II de Floridablanca. Aquellos pacientes que tuvieron una actitud po- 
sitiva ante el cepillado presentaron mayor deterioro del cepillo que los que presentaron actitud negativa. Adicionalmente, al observar la relación entre el deterioro del cepillo y la higiene oral de los adultos mayores no se encontró relación alguna. Por tanto el deterioro del cepillo no parece influenciar en el nivel de higiene oral de los adultos.

\section{BIBLIOGRAFÍA}

1. García Y, Flórez L, Silva L, Aguilar E, Concha S. Evaluación de la efectividad de un programa educativo en higiene oral dirigido a cuidadores de adultos mayores de los asilos San Antonio y San Rafael de la ciudad de Bucaramanga. Ustasalud 2006; 5:40-48.

2. Pinzón S, Zunzunegui M. Detención de necesidades de atención bucodental en ancianos mediante la autopercepción de la salud oral. Rev Mult Gerontol 1999; 9: 216 - 224.

3. Tan E, Daly C. Comparison of new and 3 - month - old toothbrushes in plaque removal. J Clin Periodontol 2002; 29: 645 - 650 .

4. Bergström J. Wear and hygiene status of toothbrushes in relation to some social background factors. Swed Dent 1973; 66: 383 - 391.

5. Glaze PM, Wade AB. Toothbrush age and as it relates to plaque control. J Clin Periodontal 1986; 13: 52-56.

6. Rawls H, Mkwayi-tulloch N, Casella R, Cosgrove R. The measurement of toothbrush sear. The Gillette Company, Boston Research \& Development, Gillette Park Boston, Massachusetts 02106-2131.

7. Sainsbury A, Seebass G, Bansal A, Young JB Reliability of the Barthel Index when used with older people. Age Ageing 2005; 34: $228-232$.

8. Moreira R, Saliba C, Dos Anjos C, et al. La influencia de la motivación y del cepillo supervisado en los hábitos de higiene de preescolares brasileños. Acta Odontol Venez 2007; 45: $534-539$.

9. Collin C, Wade DT, Davies S, Horne V. "The Barthel ADL Index: a reliability study." Int Disability Study 2009; 10: $61-63$.

10. Loewen SC, Anderson BA. Reliability of the Modified Motor Assessment Scale and the Barthel Index. Phys Ther 1988; 68: 1077 - 1081.

11. Richards SH, Peters TJ, Coast J, Gunnell DJ, Darlow MA, Pounsford J. Inter-rater reliability of the Barthel Index. Department of Social Medicine, University of Bristol. Bristol, UK, 2008.

12. República de Colombia. Ministerio de Salud. III Estudio Nacional de Salud Bucal - Ensab III Estudio Nacional de Factores de Riesgo de Enfermedades Cronicas - enfrec II. Disponible en: http://onsb.udea.edu.co/site/images/pdf/ ensab3.pdf.

13. Rangel JC, Lauzardo G, Quintana M, Gutiérrez ME, Gutiérrez N. Necesidad de crear programas de promoción y prevención en el adulto mayor. Rev Cubana Estomatol [en línea]. 2009 [fecha de acceso: enero de 2010]; 46 (1). URL disponible en: http://scielo.sld.cu/scielo.php?script = sci arttext\&pid $=$ S0034-75072009000100004\&lng $=\mathrm{es}$

14. Graveland MP, Rosema NA, Timmerman MF, Van der Weijden GA. The plaque removing efficacy of a finger brush (I-Brushs). J Clin Periodontol 2004; 31: 1084 -1087.
UnIVERSIDAd Santo Tomas PRIMER CLAUSTRO UNIVERSITARIO DE COLOMBIA B U C A R A M A N G A

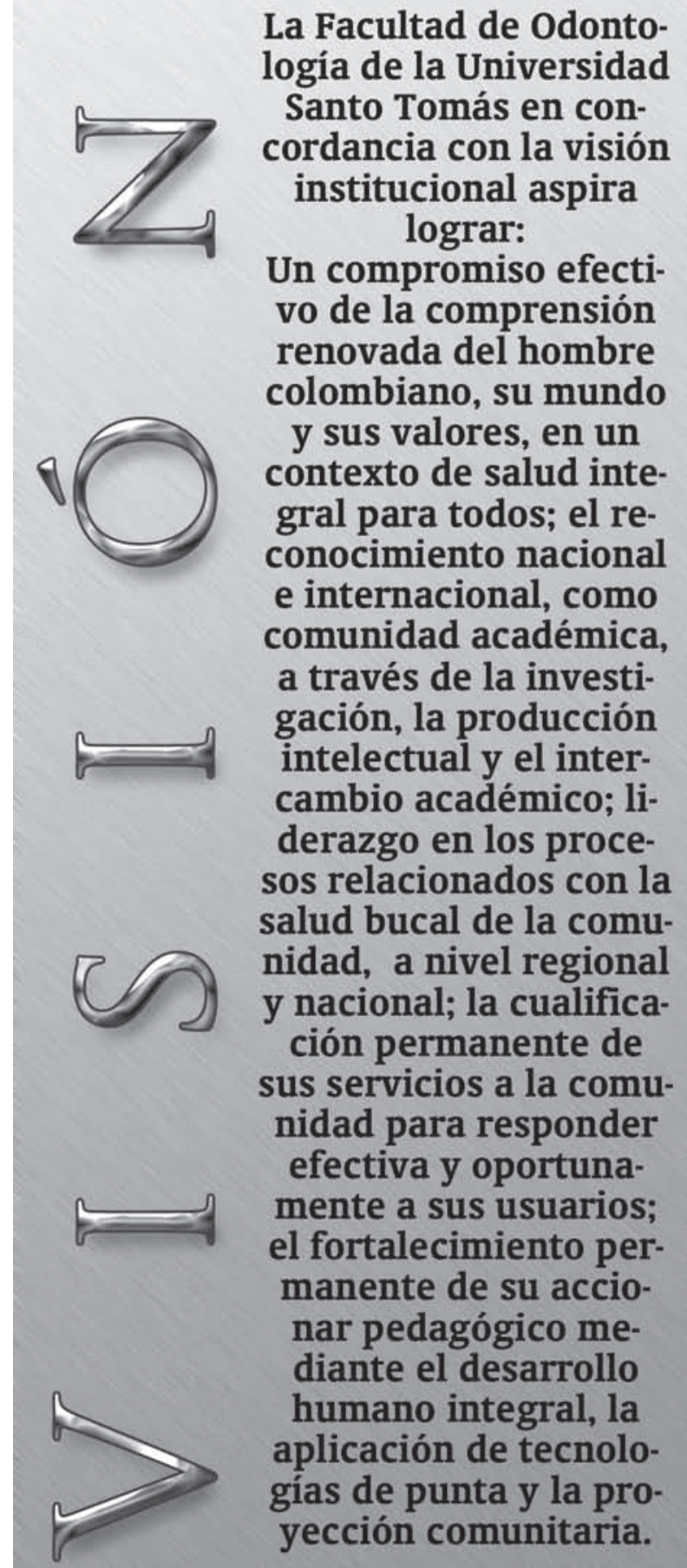

